\title{
Flexor Pollicis Longus Muscle
}

National Cancer Institute

\section{Source}

National Cancer Institute. Flexor Pollicis Longus Muscle. NCI Thesaurus. Code C150851.

A muscle that originates on the anterior surface of the radius and interosseous membrane and inserts into the palmar aspect of the base of the distal phalanx of the thumb; It is involved in flexion of the thumb and is supplied by the anterior interosseous branch of the median nerve and the radial and anterior interosseous arteries. 